has been undertaken. Physiological considerations form the starting point; organic and functional disorders are discussed in detail. "The ill wind of war has blown good to surgery and surgeons, and, through them, to coming generations" (Ogilvie ${ }^{24}$ ) some of the lessons gathered from the treatment of arterial war injuries are outlined here.

\section{REFERENCIS}

I. TREVOR, D. (1944), Proc. R. Soc. Med., 38, 76 . 2. MAKINS, G. H. (I9I9), "Gunshot Wounds in the Blood Vessels,"

3. HOLMAN, E. (1944), Surg. Gynec. Obstet., 78, 275.

Idem. (1944), Brit. Med. Bull., 2, 394.

4. LE GROS CLARK, W. E. (I945), Lancet, 1, I7.

5. HOLMAN, E. (1942), Surg. Gynec. Obstet, 75, i 83.
6. MAYBURY, B. C. (1945), Bull. War. Med., 5, 417.

7. WILSON, W. C. (1933), Brit. J. Surg., 20, 393.

8. REICHERT, F. C. (I93I), Bull. Johns Hopkins Hosp., 49, 86.

9. POWER, W. R. (1945), Brit. Med. J., 1, 656.

Io. See M.R.C. War Memo I3 "Arterial Injuries," p. 22.

II. PARKES, A. R. (1945), Brit. J. Surg., 32, 403 .

I2. COHEN, S. M. (I944), Lancet, 1 , I.

13. LEWIS, T. (1943), Proc. R. Soc. Med., 36, 522

I4. GRIFFITHS, D. L. (1940), Brit. J. Surg., 28, 239.

I5. MURRAY, G. D. W., and JANES, J. M. (1940), Brit. Med. J., 2, 6. 16. MURRAY G D. W (I940), Brit. J. Surg, 27, 567 .

77. BLAKEMORE A. H., LORD, J. W., and STEFCKO, P. L. (1942) Surgerv, 12, 488 .

Idem. (1943), Ann. Surg., 117, $48 \mathrm{I}$.

18. BLAKEMORE, A. H., and LORD, J. W. (1945), Ann. Surg., 121,

19. SHARPEY-SCHAFER, E. P. (1944), Brit. Med. Bull., 2, I7r.

20. DRUMMOND, R. (1943), Brit. Med. J., 2, 319.

2I. COURNAND, A. et al. (1943), Surgery, 13, 964.

22. BOOTHBY, W. M. (I938), Proc. Mayo Clin., 13, 64 I.

23. LEWIS, T. (194I), Brit. Med. J., 2, 795 .

24. OGILVIE, W. H. (1945), Practitioner, 155, 257.

\title{
ANAESTHESIA FOR THE SURGERY OF PERIPHERAL VASCULAR DISEASE
}

\author{
By \\ C. G. Townsend, M.A., M.B., B.Ch.(Cantab.), D.A.
}

(Specialist Anaesthetist, E.M.S., Sector X., Acting Hon. Anaesthetist, Woolwich War Memorial Hospital.)

Advances in the surgery of peripheral vascular disease during the last decade, as has been the case in many other surgical fields, have been made in close conjunction with progress in the art of anaesthesia. Whether the task before the surgeon is to discover the probable improvement in the circulation of a limb after a proposed sympathectomy or to perform a life-saving amputation on an elderly patient with arteriosclerotic gangrene and diabetes, the modern anaesthetist can provide valuable assistance.

Increasing familiarity with the physiological action and technique of local and spinal analgesia, the introduction of cyclopropane and the "closed circuit" machine in inhalation anaesthesia, and the application of limb refrigeration, both as a form of treatment and to produce analgesia, are all factors which have been of vital importance in dealing with the vascular case. Whereas an early case of thrombo-anglitis obliterans is usually a normal risk, the patient with several years' history of Beurger's disease, although appearing perfectly fit, may have unsuspected cerebral or coronary lesions and demand special care and consideration from the anaesthetist. At the other end of the scale is the diabetic arteriosclerotic with rapidly advancing gangrene of the lower limb. Such a case may appear almost moribund on reaching the theatre, but a successful operation without further deterioration in the general condition combined with the subsequent satisfactory recovery justifies the boast of the modern anaesthetist that no patient is too ill for anaesthesia if an operation may save his life.
General Anaesthesia.

The guiding principles in anaesthetising the vascular patient are of course the same as those applicable to the administration of any anaesthetio but in the presence of marked vascular disease these principles become of vital importance and mus be scrupulously observed.

The advanced case of peripheral vascular disease has a failing peripheral circulation and often a poor myocardium resulting in anoxia of the tissues. It is, therefore, essential that the anaesthetist should not'further prejudice his patient's chances of recovery by producing an anoxaemia, which would give rise to the outpouring of toxic metabolic products detrimental to both the general and local condition. Any technique which causes a sudden rise or fall of blood pressure cannot be permitted. There is likely to be a diminished hepatic and renal function and, in the case of the diabetic, ketosis. The minimum of lipoid soluble anaesthetic agents should, therefore, be used, as they themselves are thought by some to produce a histotoxic anoxiar and in addition are known to diminish the alkali reserve. ${ }^{2}$

In order to avoid the artificial production of $N$ anoxaemia the patient must breathe an amply $N$ oxygenated atmosphere, a perfect airway must be $\stackrel{\sim}{N}$ maintained, and the depth of anaesthesia should $\omega$ never be greater than will allow the surgeon to

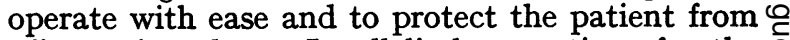
afferent impulses. In all limb operations for the $\mathscr{D}$ diseases under discussion, first plane anaesthesia (Geudel's classification) is adequate. At this level 
there is no intercostal paralysis, and the respiratory movements are sufficient to ensure a normal pulmonary ventilation and venous return. To prevent sudden variations in the blood pressure the induction should be smooth and not unduly rapid, and be followed by a level maintenance. The anaesthetist should aim at obtaining a quick return of consciousness after operation.

As Samuels with his wide experience confirms, cyclopropane is the inhalation agent of choice, ${ }^{3}$ providing as it does an atmosphere rich in oxygen, a smooth induction, light maintenance and a rapid recovery. The arterial oxygen content of patients breathing cyclopropane and oxygen mixtures has been found to be higher than when they were conscious, ${ }^{4}$ and they often leave the theatre in a better general condition than when they entered.

Nitrous oxide is a suitable agent in so far as it is non-toxic, but it may be impossible to maintain anaesthesia without anoxaemia unless there has been adequate premedication. If the anaesthetist has any doubt as to his ability to prevent suboxygenation during anaesthesia he should use the nitrous-oxide-oxygen as a vehicle for minimal amounts of trilene or vinesthene.

In spite of much that has been said and written concerning the toxicity of di-ethyl ether, and in the writer's opinion it is best avoided in the ill vascular case, there are several factors in its favour which are not always recognised. Ether anaesthesia can be maintained in combination with a more highly oxygenated atmosphere than is the case even with cyclopropane. Light ether narcosis requires only 5-7 per cent of the agent, whilst cyclopropane must be present in a concentration of at least ro per cent. In addition at a light level ether produces a peripheral vaso-dilatation and the cardiac depressant action is minimal.

Pentothal can be used to anaesthetise the vascular case, but should be given in small doses and in a concentration of not higher than 5 per cent. The injection must be made slowly in order to prevent a sudden fall of blood pressure. The writer has frequently demonstrated that if $\mathrm{I}-2$ minutes are taken over injecting 0.5 gram of pentothal in 5 per cent solution there is no appreciable fall in blood pressure, but the injection of a similar dose in less than ten seconds will temporarily lower the systolic pressure of a fit patient from IO-I5 per cent. When used as the chief agent pentothal can be given by an intravenous drip in $\frac{1}{2}-\mathrm{I}$ per cent solution. Even in good risk cases, however, a maximum dose of $\mathrm{I}_{2} \frac{1}{2}$ grams should never be exceeded as over this dose such a profound saturation with the drug occurs that, although the patient appears to have remained in the first plane throughout the operation, the full return to consciousness may be delayed for several hours. If further anaesthesia is necessary, well oxygenated nitrous oxide should be employed. When continuous pentothal is being used for an elderly or aesthenic patient with poor pulmonary $\cong$

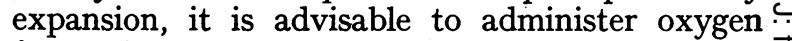
from the anaesthetic machine throughout the $\overrightarrow{\vec{F}}$ operation to prevent undue depression of the res- $\vec{\sigma}$ piratory centre and consequent anoxia. In a case $\frac{\bar{C}}{\sigma}$ where a suitable vein is difficult to find or the $\frac{\bar{\omega}}{\bar{\omega}}$ superficial veins have been thrombosed by previous $\vec{\Phi}$ intravenous injections, a pentothal drip can be given through a short sternal or tibial ${ }^{5}$ needle. क

Premedication with omnopon and scopolamine $\vec{\circ}$ before general anaesthesia should be given with $\overrightarrow{-}$ caution and in reduced dosage for the poor risk. $\vec{\sigma}$ The scopolamine may be omitted in the elderly as -0 they are very susceptible to the drug. If pentothal $\frac{0}{3}$ or cyclopropane-both depressants of the respiratory centre-are to be employed, it is often advis- N able to omit the opiate and to give atropine only. N

In selecting a general anaesthetic the condition $\vec{\sigma}$ and age of the patient together with the severity and probable duration of the operation are factors $\frac{}{2}$ which should all be taken into account. It is the writer's practice to use cyclopropane for lower limb ${ }_{\mathbb{D}}$ amputations in the medium and some poor risk 을 cases. For such operations as pre-ganglionic sym @ pathectomy in the upper limb or the repair of traumatic femoral arterio-venous fistula, pentoth $\overrightarrow{0}$ followed by nitrous oxide-oxygen, with the addition of minimal trilene if necessary, is a suitable cors bination.

\section{Spinal Analgesia.}

Spinal analgesia is of value in the diagnosis $\frac{0}{8}$ and treatment of vascular cases, but it should never be used for the arteriosclerotic with limb gangrene. 을 Such a patient is likely to have a grossly impaired coronary circulation and to risk lowering the blood pressure by means of a medium or high spinal block is to court disaster.

To produce a transient paralysis of the sympathetic supply to the lower limbs, a spinal up to Dro is preferred to a paravertebral block. Unilateral spinal analgesia has recently been tried prior $\frac{\circ}{3}$ to an arteriogram of a leg. Previously the artery was always exposed under local infiltration with I per cent procain. It is considered that a spinal $\frac{D}{0}$ block paralysing the vaso-constrictors ensures a full dilatation of the collateral vessels. Pre- N ganglionic lumbar sympathectomy is performed $N$ under a bilateral spinal. For the more severe and $\mathrm{N}$ prolonged operations it is preferable for the patient $\omega$ to be asleep. This may be achieved with a small dose of pentothal followed by nitrous oxide oxygen. $\stackrel{\circ}{\complement}$ Light inhalation anaesthesia during operations under spinal analgesia assists in the maintenance of blood pressure and aids pulmonary ventilation. 
"Heavy Nupercaine" ( $I$ in 200 in 6 per cent Glucose, sp. gr. I.024 at $I 5^{\circ} \mathrm{C}$.) is the solution employed as a routine for sub-arachnoid injection. The level of analgesia is chiefly dependant on the quantity of nupercaine used and on gravitational flow, which is controlled by positioning the patient. For "medium spinals" up to D9 an adequate dose is $\mathrm{I} \cdot 8$ c.c.

In order to render one leg analgesic the patient is kept on the affected side for five minutes after injection. The spinal column should be horizontal and to achieve this the table may require a slight cephalic tilt. If it is desired to anaesthetise both legs the patient is turned on his back immediately after the spinal injection. Both knees and hips are fully flexed for five minutes in order to eliminate the lumbo-sacral curve whilst the table remains horizontal, the aim of the anaesthetist being to permit the solution to flow equally over the sacral and lumbar roots. For a bilateral block up to D6 the above technique is followed but the table is given a cephalic tilt of $5-10^{\circ}$. Vaso-constricting drugs are not given as a routine with medium spinals. It has been shown that the anterior roots below L2 do not contain vaso-constrictor fibres, so that a block to the level of Dro does not affect the blood pressure to the same extent as do the high blocks. ${ }^{6}$ If a pressor drug is thought to be indicated Methedrine $\mathrm{mgm}$ I5 is used. The action is prolonged and compared with the other vasoconstrictors Methedrine appears more capable of raising an already fallen blood pressure. If the full dose of mgm 30 is given, the surgeon may complain of oozing in the wound. Some anaesthetists are of the opinion that a marked fall of blood pressure after spinal analgesia is of no importance, insomuch as the volume of circulating blood has not decreased. It is considered, however, that in cases of vascular disease especially, it is preferable to give a pressor drug than to allow the systolic blood pressure to remain for long below I00. When it is not intended to give a general anaesthetic in addition, the routine sedative before spinal analgesia is Nembutal grams 3 by mouth two hours before coming to the theatre. If the patient is still restless or apprehensive, omnopon gram $\frac{1}{3}$ rd can be injected intravenously.

\section{Local Analgesia.}

Local analgesia is becoming increasingly valuable to the surgical team now that blocks based on the regional anatomy are tending to replace the more indiscriminate infiltration of the part, generally referred to as a "spot of local."

For all operations on the upper limb a brachial plexus block is the anaesthetic of choice. Prominence has recently been given to various tech- niques ${ }^{9}, 10$, for stellate ganglion block in order to paralyse the cervical sympathetic, but it should $\frac{2}{3}$ be noted that blocking the brachial plexus as it $\stackrel{\otimes}{\circ}$ crosses the first rib includes the vaso-constrictors $c$ and gives full dilatation of the brachial vessels. In addition after some 50 per cent of brachial blocks $\stackrel{\oplus}{\stackrel{9}{\oplus}}$ Horner's syndrome may be observed. ${ }^{7}$ This is attributed to the solution tracking up to the 음 ganglion, which has a communication with each $\frac{\omega}{\sqrt{0}}$ nerve making up the brachial plexus.

The method used to obtain a brachial block is that first worked out by Patrick ${ }^{8}$ and recently so lucidly described by Macintosh and Mushin. ${ }^{7} ?$ The object of the technique is to flood the upper $\overrightarrow{\vec{\omega}}$ surface of the first rib immediately postero-lateral $\stackrel{\circ}{\circ}$ to the subclavian artery. The whole of the plexus 8 in the region is thus included and the obtaining 3 of paraesthesias at the first needling are not essen- N tial though satisfactory to the operator. The i needle is entered half an inch behind the mid point $\stackrel{f}{A}$ of the clavicle and is directed downwards, inwards and backwards on to the first rib. The pulsation 0 of the subclavian artery, with which the shaft of the needle may well be brought in contact, provides the final localisation. The writer uses a solution $\mathbb{O}$ containing equal parts of I per cent procain and I: 2,000 nupercain with $I: 250,000$ adrenalin, whic provides an analgesia up to three hours' durationg A volume of 60 c.c. should be used unless paraes thesia is obtained on needling, when 30 c.c. wi suffice. In old or asthenic patients a half strength is adequate, but the same volume must be used. It is important to have an assistant to depress the shoulder by pulling on the arm in order to expose the more medial cord of the plexus from beneath $\propto$ the subclavian artery. It has been observed that $\overrightarrow{\vec{A}}$ using I per cent procain a sensory loss only is 3 obtained, but that with 2 per cent procain a motor paralysis develops. This point is of value when it is desirable for the patient to make voluntary movements at the request of the surgeon during 3 operation. It must not be forgotten in this con- $\bar{\sigma}$ nection that the presence of a tourniquet round the arm will produce a motor paralysis after half $ᄋ$ an hour due to nerve asphyxia. The danger of causing gangrene of a finger or toe following 은 anaesthesia with a digital nerve block is becoming well known ${ }^{11}$ and ${ }^{12}$, but this risk is much higher in the presence of vascular disease. If it is, however, considered essential to employ a local digital o block the following precautions should be taken ${ }^{7}$

(I) The anaesthetic solution must not contain adrenalin.

(2) The injections must be made at two different $\stackrel{\circ}{\frac{C}{D}}$ levels on each side of the digit in order to avoid forming a constricting collar of fluid.

(3) A digital tourniquet must not be used. 
Operations on the leg and foot can be satisfactorily carried out under local nerve block. The common peroneal nerve is easily accessible where it lies on the back of the head of the fibula and ro c.c. of 2 per cent procain with I:250,000 adrenalin are injected in this region. The tibial nerve can be blocked at the distal end of the popliteal fossa where it lies on the medial side of the popliteal vessels and 20 c.c. of the local solution are employed. Efferent and afferent fibres are paralysed and a full dilatation of the vessels of the foot is assured. Arterectomy is usually carried out under infiltration with $\frac{1}{2}$ per cent procain. Embolectomy should also be performed under local infiltration. ${ }^{13}$ Owing to the tendency of the embolic case to develop anuria it is especially important to avoid anaesthesia with the more toxic general agents.

The local injection of procain around the damaged vessels has been recommended in the treatment of traumatic arterial spasm, but this procedure is now thought to be ineffective. ${ }^{14}$ Cohen observes that the paralysis of the main local nerve in the region of the artery causes cutaneous dilatation. The consequent improvement in the warmth and colour of the affected limb gives a false impression of the deep vessel spasm having been overcome.

\section{Refrigeration Anaesthesia.}

During the last three years there has been an increasing appreciation of the value of cold in the treatment of certain vascular injuries and diseases. The scope of cold therapy both in range and degree of application is extensive. Gentle cooling of the Ischaemic limb to decrease metabolic needs during the recovery of the collateral circulation is recommended. ${ }^{15}$ Refrigeration of the crushed or gangrenous limb relieves pain, stops the output of toxic metabolites and provides complete anaesthesia prior to amputation of the part. By refrigeration is meant not freezing but reduction of temperature to between $15^{\circ}-5^{\circ} \mathrm{C}$. Below $15^{\circ} \mathrm{C}$. there is complete anaesthesia whilst enzyme action and the growth of organisms are suspended. Amputations under refrigeration can be successfully performed in desperately ill cases of diabetic, arteriosclerotic and embolic gangrene, which might otherwise be deemed inoperable. Icing of the recently crushed limb is said to prevent muscle autolysis and renal damage. ${ }^{16}$ In favour of the method are the absence of pain, shock, or fall in blood pressure during or after operation, together with a marked freedom from post-operative pulmonary complications. ${ }^{17}$ The disadvantages include, the length of time required to produce anaesthesia, the use of from seventy to one hundred pounds of ice for each case, some delay in healing, and a small risk of infection from organisms in the ice. ${ }^{18,19}$ This latter will be eliminated by the use of electrically controlled limb $\frac{0}{\vec{N}}$ refrigerators, which are now becoming available. For the cases at which the writer has assisted the technique originally described by F. M. Allen $\stackrel{2}{C}$ has been followed. $20,21,22$

Premedication of omnopon gram $\frac{1}{6}$ th $-\frac{1}{3}$ rd, prior $\vec{F}$ to refrigeration, is given to all except the stoical or very ill patient. This dose may be repeated $\frac{}{\circ}$ intravenously just before entering the theatre. $\overline{\bar{w}}$. The tourniquet level is chilled by ice bags for half $\bar{\phi}$ an hour before the application of two superimposed turns of half-inch rubber tubing, $3-4 \stackrel{ }{\text { क }}$ inches above the the proposed saw line. The $\overrightarrow{0}$ limb is then encased in chipped ice and a rubber $\overrightarrow{.}$ sheet to a level two inches above the tourniquet. $\vec{\omega}$ Raising the head of the bed on blocks allows ther melted ice to drain off the end of the macintosh 3 sheet into a bucket. In the case of an arm ori below the knee amputation the limb may hang into N a bucket of ice and water. Two and a half hours is the minimum time required to produce anaes- $-\vec{S}$ thesia for amputation through the thigh, but the $\mathrm{V}$ time in each case varies with the diameter of thes limb and the patency of the blood vessels. During $\overrightarrow{-}$ refrigeration the patient dozes, reads or knits. The ${ }^{\circ}$ upper part of the body is kept well covered and욷 warm drinks are given to maintain the normal bodyg⿳亠丷厂彡⿱ temperature. This is not difficult as there is no conduction of cold from below the level of tgee tourniquet. It is important that diabetics shoufdo continue their normal treatment and full diet to the time of operation.

The ice should not be pressed tightly against the skin or local blistering may follow, but must, how-券 ever, bo packed well under the thigh or anaesthesia $\frac{}{\circ}$ of the sciatic nerve may be incomplete. In two $\Rightarrow$ cases where this deficiency occurred there was some을 permanent knee flexion preventing full extension of the leg. When during operation there was a complaint of pain on reaching the sciatic the sur-geon injected 5 c.c. of 2 per cent procain into the 3 . nerve. Section then appeared painless. In all:other cases the patients seemed to be entirely?. unaware of the progress of the operation. Fromo $\frac{1}{2}-\mathrm{I}$ hour's complete anaesthesia is obtained. The patient's ears are packed with cotton wool ando during the sawing of the bone diversionary noises? are made, e.g. running of all the theatre watero taps. After operation the stump is chilled by three ice bags laid against light dressings. This is con- $-\widetilde{O}$ tinued for seventy-two hours, the temperaturen being gradually raised by applying heavier dres-N sings daily. This procedure minimises the amount $t^{\omega}$ of blood needed by the flaps. Though healing is slowed-the stitches should not be removed fore fourteen days-union is usually primary and theres is less sloughing and infection than occurs in similar types of cases not treated by refrigeration. 


\section{Summary.}

Indications for the use of the different methods of anaesthesia, together with their limitations in cases of peripheral vascular disease are discussed. The technique of refrigeration anaesthesia is fully described.

\section{BIBLIOGRAPHY}

I. PASK, E. A. (July 104I) Brit. J. of A maesthesia, 129 et seg. 2. HEWAR, C. LANGTON, "Recent Advances in Anaesthesia," 5th edition, 3 .

3. SAMUELS, S. S., "Diagnosis and Treatment of the Diseases of the Peripheral Arteries," 258.

4. SMITH, C. A. (1939), Surgery, Gymae, and Obstet., 69, 584.

5. GIBBONS, LESLIE A. (June 23, 1945), Brit. Med. Jourmal, 878.
6. HARRIS, T. A. B. (July I939), Brit. J. of A raesth., 136.

7. MACINTOSH and MUSHIN, "Local Anaesthesia: Brachial Plexus,"

8. PATRICK, J (1940), Brit.J. Surgery, 27, 734-739.

9. VOLPITTO and RISTEEN (Sept. 1944), "Anaesthesiology," 491.

ro. M. R. C. (1944), "Arterial Injuries, Early Diagmosis and Treatment," 22.

r. HEWAR, C. LANGTON (1944), Medical Anmual, 26.

12. SAMUELS, S. S., "Diagnosis and Treatment of Diseases of the Peripheral Arteries," 320

13. M. R. C. (1944), "Arterial Injuries," 37.

14. COHEN, S. M.' (Jan. I, 1944), Lancet, 2 .

15. M. R. C., "Arterial Injuries, Early Diagnosis and Treatment," I 8.

I6. COHEN, S. M. (Jan. I, I944), Lancet, 6.

17. COHEN, S. M. (March I944), Proc. Royal Soc. Med., 232.

18. KING. E.-Brit. Med. J., 1944., 2, 662.
19. CAYFORD, E. A. and PRETTY, H. G (1945) Ann. Surgery, 121, 157. 20. ALLEN, F. M. (Jan.-Feb. 1943), "Anaesthesia and Analgesia.", 2I. ALLEN, F. M. (Sept.-Oct. 1944), "Anaesthesia and Analgesia." 22. ALLEN, F. M. (Mar.-Apr. I945), "Anaesthesia and Analgesia."

\section{LITERARY SECTION}

\section{THE 1945 ASLIB CONFERENCE}

Aslib (Association of Special Libraries and Information Bureaux, 52, Bloomsbury Street, W.C.I), born in I924, celebrated its coming of age at a most successful annual conference, held under the Chairmanship of Sir Frederic Kenyon at Portland Hall on September I5 and I6, I945. Having among its objects the co-ordination and systematic use of sources of knowledge and information in the arts, sciences; industry, and commerce, its activities include an annual meeting, the maintenance of an enquiry bureau and of a register of expert translators, and the publication of books, book-lists, and journals. The year I944-45 was a memorable one in the history. of Aslib, as for the first time the receipt of a Government grant marked official recognition of its work.

\section{A Revolutionary Proposal}

The usual presidential address was replaced by a fascinating and provocative paper, "Information Service as an Essential in the Progress of Science," by J. D. Bernal, F.R.S., University Professor of Physics at Birkbeck College, which dealt primarily with the user's side of library service in relation to research. In the past, said the lecturer, the world of the scientific research worker and the world of the librarian had been too far apart. Old libraries were conceived as depositories of knowledge. The modern library should be a distributor and organiser of knowledge. While the annual increment of new knowledge in any particular field of science was rapidly growing, the capacity of the individual research worker for assimilating knowledge had already, in many cases, reached its limits. It was the librarian's duty not merely to accept material for his library, but to insist on its presentation in a form suitable for distribution. This meant a more intimate relationship between librarian and research worker and between librarian and publisher, both of periodicals and of books.

The primary unit in scientific publication, according to Professor Bernal, was the scientific paper dealing comprehensively with a particulàr topic. Books could be considered as an accumulation of papers, and notes and letters as papers in embryo. The organisation of publication and recording should therefore centre on that of the scientific paper. In the lecturer's opinion the journal had ceased to be a satisfactory means \& distributing scientific information. The majorit. of scientific journals were produced for learneg societies, in the case of the smaller ones, principally for exchange purposes.

In Professor Bernal's proposed scheme each country would have a centre of scientific publica- $\stackrel{\mathbb{Q}}{\varrho}$ tion and exchange which would receive from $\vec{F}$ societies' papers already passed by referee sas 윽 suitable for publication and lists of members to whom papers on specified subjects from any part of the world should be sent. These national centres would thus be clearing houses for scientific 3 papers, arranging internal distribution and, at $\dot{0}$ the same time, sending copies to clearing houses 3 in other countries. Membership subscription to $\delta$ one society would entitle the subscriber to the services of the whole organisation, which, inci- o dentally, could make a very good claim for government support. Papers would possess uniform format, so that they could be conveniently bound according to subject. A similar service os could be organised for science abstracts, prepared $N$ once and for all by the author, verified by his own N scientific society, and produced in a form suitable $\sigma$ for modern filing systems. Similarly the scientific societies could make themselves responsible for $\stackrel{\varrho}{=}$ the publication of monographs and textbooks, $\stackrel{\Phi}{\oplus}$ allowing freedom, however, for original work of ? an unorthodox kind. 